\title{
Prevalencia del Consumo de Alcohol en Traumatizados Atendidos Cali, Colombia
}

\author{
1Juan Calle, ${ }^{2}$ Álvaro Sánchez, ${ }^{3}$ Alberto García, ${ }^{4}$ Mónica Morales
}

\section{RESUMEN}

Introducción: La ingesta alcohólica se asocia con riesgo de trauma y reincidencia. La información local es escasa. Se determinarán la prevalencia del consumo de alcohol y las características asociadas, en traumatizados atendidos en dos hospitales de Cali, Colombia.

Métodos: Estudio observacional analítico del registro de trauma de dos hospitales de Cali, Colombia, entre enero y diciembre de 2012.

Se incluyeron sujetos de 14 años o más, con lesiones de causa externa violenta, accidental, autoinflingida o por tránsito. Se excluyeron embarazadas, reclusos o personas con lesiones por agentes químicos, ambientales, o tóxicos. Se efectuó un análisis univariado y multivariado de variables asociadas con la probabilidad de reporte positivo de alcohol.

Resultados: Se incluyeron 10290 pacientes, 63.4\% hombres. El trauma contuso fue más frecuente (59\%), seguido por tránsito (12\%), arma blanca (16\%) y arma de fuego (9\%).

El reporte de ingesta alcohólica fue más frecuente en: hombres (OR 6.88 I.C. 95\% 3.17-4.9), lesionados durante la madrugada, (OR: 7.54 IC 95\% 6.43-8.84) pacientes no asegurados, (OR 3.31, IC 95\% 2.69-4.08), individuos entre 20 y 44 años (OR 1.61 IC 95\% 1.39-1.85), pacientes con ISS $\geq 9$, (OR 2.58, IC 95\% 2.18-3.05), lesiones por tránsito o mecanismos penetrantes (OR 4.46, IC 95\% 3.86-516), lesiones no relacionadas con el trabajo $23.68(16.1-34.8)$ y atención en el hospital público 12.66 (10.46-15.32).

Las asociaciones con la falta de aseguramiento y con ISS $\geq 9$ desaparecieron en la RLM.

\footnotetext{
${ }^{1}$ Estudiante de Medicina, ${ }^{2}$ Residente de Cirugía General ${ }^{3}$ Profesor Asociado, Escuela de Medicina, Facultad de salud ${ }^{4}$ Estadística

${ }^{1}$ Departamento de Medicina, Universidad ICESI, Cali, Colombia

${ }^{2}$ Departamento de Cirugía, Universidad CES, Medellín, Colombia

${ }^{3}$ Departamento de Cirugía, Universidad del Valle, Cirugía de Trauma y Emergencias, Cuidados Intensivos, Fundación Valle del Lili, Cali, Colombia

${ }^{4}$ Registro de Trauma, Universidad del Valle, Cali, Colombia

Corresponding Author: Alberto García, Profesor Asociado Escuela de Medicina, Facultad de Salud, Departamento de Cirugía Universidad del Valle, Cirugía de Trauma y Emergencias Cuidados Intensivos, Fundación Valle del Lili Colombia, e-mail: alberto.garcia@correounivalle.edu.co
}

Conclusiones: Encontramos una prevalencia mayor de consumo de alcohol en los lesionados en la madrugada, hombres, adultos de edad media, víctimas de trauma por tránsito o penetrante, lesiones no relacionadas con el trabajo y atención en el hospital público.

Palabras claves: Alcohol/abuso de sustancias, Control de trauma, Heridas y lesiones, olitraumatismo, Prenención del trauma.

How to cite this article: Calle J, Sánchez Á, García A, Morales M. Prevalencia del Consumo de Alcohol en Traumatizados Atendidos Cali, Colombia. Panam J Trauma Crit Care Emerg Surg 2015;4(2):77-86.

Source of support: Nil

Conflict of interest: None declared

\section{ABSTRACT}

Introduction: Alcohol intake is associated with risk for trauma, and recidivism. The local information is scarce. This study evaluates the prevalence of alcohol and associated features among trauma patients in two hospitals in Cali, Colombia.

Materials and methods: Analytical observational registry trauma of two hospitals in Cali, Colombia, between January and December 2012. Subjects aged 14 or more were included. We excluded pregnant prisoners or people with injuries from chemical, environmental, or toxic agents. Univariate and multivariate analysis of variables associated with the probability of reporting positive for alcohol.

Results: The 10,290 patients were included, $63.4 \%$ were men. Blunt trauma was most common $(59 \%)$, followed by traffic crashes (12\%), stab (16\%) and firearms (9\%). Alcohol consumption was more frequent in: men (OR 6.88, 95\% Cl 3.174.9), injured during $\mathrm{AM}$, (OR: $7.54 ; 95 \% \mathrm{Cl} 6.43-8.84$ ) patients insured, (OR 3.31, 95\% Cl 2.69-4.08), among individuals 20 and 44 years (OR $1.6195 \% \mathrm{Cl} 1.39-1.85)$, patients with ISS $\geq 9$ (OR 2.58, 95\% Cl 2.18-3.05), or traffic injuries penetrating mechanisms (OR 4.46, 95\% Cl 3.86-516), injuries not work-related 23.68 (16.1-34.8) and in public hospitals 12.66 (10.46-15.32). Partnerships with the lack of insurance and ISS $\geq 9$ disappeared in the multivariate regression.

Conclusion: We found a higher prevalence of alcohol consumption in the injured at dawn, men, middle-aged adults, victims of trauma transit or penetrating injuries unrelated to work and care in the public hospital.

Keywords: Alcohol/substance abuse, Control trauma, Wounds and injuries.

Note: Paper presented in Student Research Competition at the Annual Congress of Panamerican Trauma Society, Panama City, Panama, November 2015. 


\section{INTRODUCCIÓN}

En el mundo 16000 personas mueren diariamente por causas traumáticas, y más del 90\% de estas muertes ocurren en países de bajo y mediano desarrollo. ${ }^{1}$

La carga que representa el trauma no se debe solo a la mortalidad que genera, sino también a la alta carga en morbilidad que genera; ${ }^{2}$ en algunos países llegando a ocupar el $30 \%$ de las incapacidades laborales, ${ }^{3}$ lo que convierte al trauma en general como un problema de salud publica. ${ }^{2}$

El comportamiento de las causas de mortalidad debida a lesiones traumáticas difiere según el grado de desarrollo del país. ${ }^{4}$ Por ejemplo, los eventos de transito ocupan la primera causa de mortalidad en los países de desarrollados, mientras que en los países en vía de desarrollo son las lesiones adquiridas por violencia interpersonal. ${ }^{4}$

En Colombia los traumatismos intencionales y no intencionales generan el 33\% de las consultas en los servicios de urgencias de todo el país, además causaron 1 de cada 3 muertes en los últimos 10 años, ${ }^{5,6}$ siendo la causa violenta la primera, aunque viene con tendencia a la disminución, acompañándose con un aumento concomitante en las causas relacionadas con lesiones relacionadas con eventos de transito. ${ }^{5,6}$

Según el Instituto Nacional de Medicina Legal, el impacto en los años de vida potencialmente perdidos (AVPP) fueron de 969,150 años para el 2012, del cual el $70 \%$ se debió a trauma relacionado con violencia y $20 \%$ relacionado con eventos de transito. ${ }^{6}$

El consumo de alcohol en el mundo, en Las Américas, en Sudamerica y en países latinos de Norte América como México, se ha asociado en general con el trauma violento y el relacionado con eventos de transito, debido a que propicia comportamientos agresivos y afecta la coordinación de las personas. ${ }^{7-9}$ Un ejemplo es que 1 de cada 3 lesiones intencionales, 1 de cada 4 lesiones no accidentales, $24 \%$ de los homicidios, $11 \%$ de suicidios y $20 \%$ de eventos de tránsito se han relacionado con el consumo de alcohol. ${ }^{10}$

En Colombia, el alcohol es la sustancia psicotóxica de mayor uso. Se sabe que más de $90 \%$ de la población adulta Colombiana consume con cierta frecuencia algún tipo de bebida alcohólica. ${ }^{11} \mathrm{El}$ patrón de consumo asociado es de tipo problemático, y el $15 \%$ se encuentran a riesgo de dependencia. ${ }^{12,13}$

El consumo de alcohol en Colombia además es aceptado y socialmente permitido, a pesar de sus consecuencias perjudiciales. ${ }^{14}$ A pesar de que se tiene evidencia suficiente que promueve el control sobre el consumo de alcohol, y existen programas relacionados con la prevención y control en este ámbito, los datos oficiales reportan un aumento en el consumo durante la última década. ${ }^{15}$

En este contexto, el alcohol juega un papel muy importante, pues desde hace varias décadas se ha conocido que el consumo de alcohol facilita la expresión de comportamientos imprudentes, de tendencias violentas, de alteraciones sensomotoras, ${ }^{16}$ que incrementan el riesgo de trauma interpersonal, personal o vehicular, ${ }^{7,17-24}$ con el agravante de que la severidad del trauma sufrido tiende a ser mayor en las personas que han consumido alcohol comparado con las que no. ${ }^{17-21}$

Teniendo en cuenta esta asociación se ha estudiado que el evento traumático podría considerarse un marcador de dependencia al alcohol, ${ }^{19}$ y que una vez se sufre una lesión relacionada con el consumo alcohólico, el paciente presenta un mayor riesgo de volver a consultar por trauma. ${ }^{19,25-31}$

La evidencia actual orienta a la búsqueda activa en forma de tamización del consumo de alcohol. Se ha identificado que hasta el $46 \%$ de los pacientes en el servicio de trauma podrían haber ingerido alcohol, y que el evento traumático podría servir como oportunidad para realizar intervenciones en relación con el consumo de alcohol. ${ }^{19,25-28,32}$

Gentillelo et al demostró que las intervenciones de tipo motivacional realizada en el servicio de urgencias en los pacientes que han sufrido trauma relacionado con consumo de alcohol son efectivas. Los pacientes con consumo leve a moderado de alcohol, mostraron una disminución del 83\% para el consumo de alcohol, del $47 \%$ para las consultas de urgencias y de un $48 \%$ para las hospitalizaciones. ${ }^{32}$ Estudios más recientes del mismo autor demuestran que la intervención realizada en el servicio de urgencias es además costo efectiva. ${ }^{32,33}$

En Cali, la magnitud de esta asociación en nuestro medio es casi completamente desconocida. En 1997 Pérez M y García A, ${ }^{34}$ reportaron positividad de la alcoholemia en $25 \%$ de 501 fallecimientos por trauma en un periodo de 4 meses del año 1995. En 2001 García A y colaboradores, encontraron la realización de alcoholemia en $83 \%$ de muertos por trauma durante 1998, el examen fue positivo en $22.5 \%$ de los casos, con una mayor proporción en los grupos de agresión por arma blanca y golpes y en lesiones de tránsito de ocupantes de automóvil y motocicleta. Bejarano M y Rendón LF encontraron antecedente de consumo de alcohol en $10.6 \%$ de pacientes traumatizados en un servicio de urgencias. La proporción fue progresivamente mayor en mayores de 13 años y se asoció con violencia intencional. ${ }^{35}$ Calle-Toro y colaboradores reportaron antecedente de ingesta alcohólica en 22.4\% de los lesionados por tránsito en un hospital público y en $5.6 \%$ de los atendidos en un hospital privado. ${ }^{36}$ 
Tabla 1: Características de los pacientes incluídos

\begin{tabular}{|c|c|c|}
\hline & \multicolumn{2}{|c|}{$\begin{array}{l}\text { Total de Pacientes } \\
\quad(N=10290)\end{array}$} \\
\hline \multicolumn{2}{|l|}{ Datos Sociodemográficos } & \\
\hline \multicolumn{3}{|l|}{ Edad en años } \\
\hline Rango & 14-89 & \\
\hline Mediana $\left[R I Q^{*}\right]$ & $32[23-47]$ & \\
\hline $20-44$ & 4441 & $43.2 \%(42.2-44.1)$ \\
\hline$<20$ o $>44$ & 5849 & $56.8 \%(55.9-57.8)$ \\
\hline \multicolumn{3}{|l|}{ Género (n, \% IC 95\%**) } \\
\hline Masculino & 7034 & $68.3 \%(67.5-69.3)$ \\
\hline Femenino & 3256 & $31.7 \%(30.7-32.5)$ \\
\hline \multicolumn{3}{|l|}{ Mecanismo de trauma (n, \% IC 95\%) } \\
\hline Arma de Fuego & 890 & $8.7 \%(8.1-9.1)$ \\
\hline Arma Blanca & 1611 & $15.7 \%(15.0-16.4)$ \\
\hline Tránsito & 1206 & $11.7 \%(11.1-12.3)$ \\
\hline Contundente & 6023 & $59.2 \%(58.3-60.2)$ \\
\hline Otros contundentes & 560 & $5.4 \%(5.0-5.9)$ \\
\hline Atención en el Hospital Público & 3874 & $37.7 \%(36.7-38.6)$ \\
\hline Ingreso entre $12 \mathrm{AM}$ y $6 \mathrm{AM}$ & 909 & $8.8 \%(8.3-9.4)$ \\
\hline Sin seguridad Social (n, \% IC 95\%) & 569 & $5.5 \%(5.1-6.0)$ \\
\hline Evento no relacionado con el trabajo & 6377 & $62.0 \%(61.0-62.9)$ \\
\hline \multicolumn{3}{|l|}{ Información Clinica } \\
\hline \multicolumn{3}{|l|}{ ISST } \\
\hline Rango & $1-75$ & \\
\hline Mediana [RIQ] & $1[1-4]$ & \\
\hline ISS < $9(n, \%$ IC 95\%) & 9095 & $88.4 \%(87.8-89.0)$ \\
\hline ISS $\geq 9(n, \%$ IC 95\%) & 1195 & $11.6 \%(11.0-12.2)$ \\
\hline \multicolumn{3}{|l|}{ RTS§ } \\
\hline Rango & $0-7.8$ & \\
\hline Mediana [RIQ] & $7.8[7.8-7.8]$ & \\
\hline \multicolumn{3}{|l|}{ Desenlaces } \\
\hline Ingesta de alcohol positiva (n, \% IC 95\%) & 928 & $9.0 \%(8.5-9.6)$ \\
\hline Hospitalización (n, \% IC 95\%) & 3047 & $29.6 \%(28.7-0.5)$ \\
\hline Cirugía (n, \% IC 95\%) & 1870 & $18.2 \%(17.4-18.9)$ \\
\hline Muerte (n, \% IC 95\%) & 158 & $1.5 \%(1.3-1.8)$ \\
\hline
\end{tabular}

${ }^{*} \mathrm{RIQ}$, Rango inter-cuartílico; ${ }^{* *} \mathrm{IC} 95 \%$, Intervalo de confianza del 95\%; ${ }^{\top} \mathrm{ISS}$, Injury severity score; ${ }^{\S} \mathrm{RTS}$, Revised trauma score

En la presente investigación se analizan la prevalencia deingesta alcohólica y los factores asociados con una mayor exposición en dos centros de trauma en Cali, Colombia, con el fin de obtener información útil para implementar estrategias preventivas en este grupo de pacientes.

\section{MATERIALES Y MÉTODOS}

Se revisó el registro de trauma del Hospital universitario del Valle y la Fundación Valle del Lili, en Cali, Colombia, desde el 1 de Enero hasta el 31 de Diciembre del 2013. Los datos fueron transferidos para su análisis al paquete estadístico STATA $^{\mathrm{TM}} 12$ (StataCorp, Texas-USA).

Se incluyeron pacientes mayores de 14 años, con lesiones de causa externa por causa violenta, accidental, autoinflingida o relacionada con tránsito. Se excluyeron mujeres en embarazo, personas recluídas en la cárcel o lesiones de causa externa ocasionadas por la exposición de agentes químicos, ambientales, o tóxicos. Fueron excluídos también los sujetos con el mecanismo apropiado en quienes no se registraron lesiones $($ ISS $=0$ ).

Se tabularon la edad, el genero, el día de la semana, la hora, el lugar en el cual se presentó, el mecanismo y tipo de trauma involucrado, la región anatómica comprometida, severidad del trauma medida con los puntaje de RTS (puntaje de trauma revisado) e ISS (puntaje de severidad de las lesiones), necesidad de hospitalización general y en UCI, necesidad de cirugía, mortalidad y hospital donde fue atendido: Fundación Valle del Lili, hospital privado de 550 camas que denominaremos Hospital 1 y Hospital Universitario del Valle, Hospital público de 770 camas, que denominaremos Hospital 2.

Análisis estadístico: Las variables continuas son presentadas como rangos y promedio-desviación estándar o mediana-rango intercuartílico, de acuerdo con su distribución normal o no.

Las variables categóricas son presentadas como proporciones e IC de 95\%. 
Tabla 2: Variables asociadas con una mayor probabilidad de ingesta alcohólica

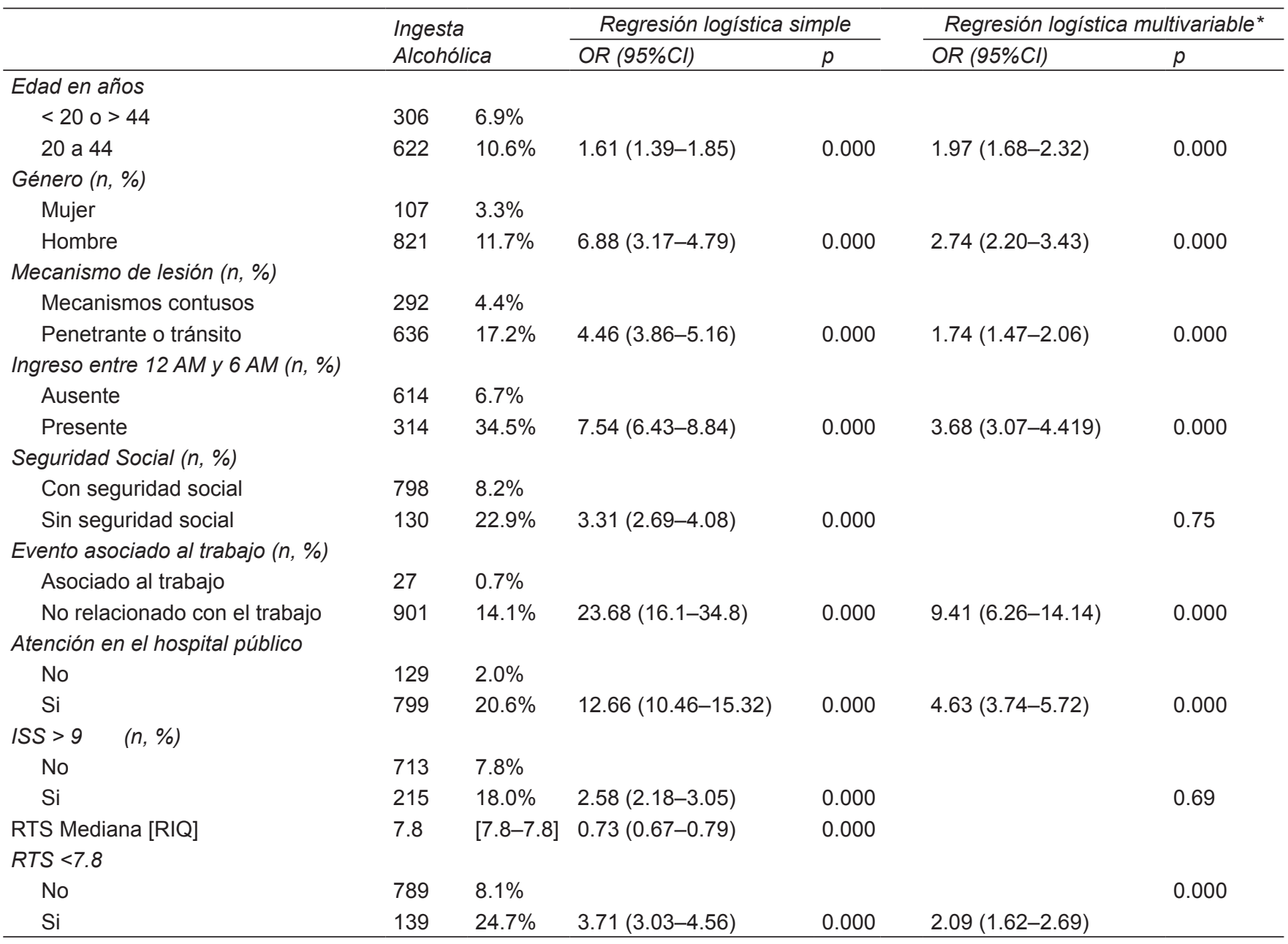

* Regresión logística multivariable con eliminación hacia atrás. Variables con $p<0.1$ fueron conservadas en el modelo.

$\mathrm{OR}$, odds ratio; $\mathrm{Cl}$, Intervalo de confianza; RIQ, rango inter-cuartílico

Para el análisis de las asociaciones la edad y en ISS fueron categorizadas. La asociación entre las diferentes variables y el reporte de ingesta alcohólica se hizo de manera univariada mediante la prueba de Chi 2. Las variables identificadas con una asociación significativa $(p<0.1)$ fueron analizadas mediante regresión logística multivariada (RLM), con retiro gradual de las variables, manteniendo en el modelo aquellas con una $\mathrm{p}<0.1$.

\section{RESULTADOS}

Fueron atendidos durante 2012, 15466 pacientes, de los cuales se excluyeron 3253 por edad < 14 años, 48 por mecanismo de lesión, 467 por ausencia de lesiones traumáticas al examen y 1408 por falta de información acerca de ingesta alcohólica.

Los 10290 sujetos restantes constituyen la base del presente análisis. 7034 eran hombres (68.4\%, IC 95\% 67.5 -69.3). La edad fluctuó entre 14 y 89 años (mediana 32, RIQ 23-47).

Los mecanismos de trauma más comunes fueron los contusos (58.5\%, IC 95\% 57.6-59.5), lesiones por tránsito
(11.7\%, IC 95\% 11.1-12.3), heridas por arma blanca (15.7\%, IC 95\% 15.4-26.4) y heridas por arma de fuego (8.7\%, IC 95\% 8.1-9.2) (Tabla 1).

La información sobre aseguramiento se describió en 10093 casos (98.1\%). En la mayoría (94.4, IC 95\% 93.9-94.8) (Tabla 1).

Es ISS fluctuó entre 1 y 75, con una mediana de 1 y un RIQ entre 1 y 4. Presentaron un ISS de 9 o más 1195 pacientes (11.65\% IC 95\% 11.0-12.2) (Tabla 1).

El RTS osciló entre 0 y 7.8 con una mediana de 7.8, y un RIQ 7.8-7.8 (Tabla 1).

Se registró ingesta de bebidas alcohólicas en 928 pacientes (9.0\%, IC 95\% 8.5-9.6). Esta fue reportada con mayor frecuencia en el Hospital 2: 20.6\%, IC 95\% 19.4-21.9 versus 2.0, IC 95\% 1.7-2.4 (Tabla 1).

\section{ANÁLISIS DE LAS ASOCIACIONES}

En el análisis univariado los hombres mostraron una prevalencia mayor de ingesta alcohólica (OR 6.88 I.C. 95\% 3.17-4.9). Un hallazgo semejante evidenciaron los individuos lesionados durante la madrugada, (OR: 7.54 
IC 95\% 6.43-8.84). Los pacientes no asegurados también mostraron una asociación significativa (OR 3.31, IC 95\% 2.69-4.08) (Tabla 2).

Los pacientes menores de 20 años y los mayores de 44, tuvieron una prevalencia baja de consumo alcohólico, a favor de los individuos entre 20 y 44 años (OR 1.61 IC 95\% 1.39-1.85).

El análisis de la relación con el RTS como variable continua mostró una reducción de la probabilidad de haber sido expuesto al alcohol en la medida que el puntaje fisiológico era más alto (OR 0.73, IC 95\% 0.67-0.79) (Tabla 2). La categorización de esta variable mostró un incremento de la exposición a alcohol entre quienes tuvieron alteraciones del puntaje (OR 3.71 IC 95\% 3.034.56) (Tabla 2).

La asociación con la severidad del trauma no fue muy intensa. La mayor prevalencia ocurrió en los pacientes con ISS $\geq 9$, (OR 2.58, IC 95\% 2.18-3.05) (Tabla 2).

Los lesionados por tránsito o por mecanismos penetrantes presentaron una asociación positiva (OR 4.46, IC 95\% 3.86-516), igual que quienes sufrieron sus lesiones en eventos no relacionados con el trabajo 23.68 (16.1-34.8). Quienes recibieron atención en el hospital público reportaron más frecuentemente consumo de alcohol (OR 12.66 IC 95\% 10.46-15.32) (Tabla 2).

En el modelo de RLM, persistieron las asociaciones encontradas con el grupo etáreo de 20 a 44 años, con el género masculino, la hora del trauma, las lesiones no relacionadas con el trabajo, la alteración fisiológica y con la atención en el Hospital 2.

Las asociaciones con la falta de aseguramiento y con ISS $\geq 9$ desaparecieron en la RLM (Tabla 2).

Requirieron hospitalización 3047 pacientes, cirugía 1870 y 158 fallecieron.

\section{DISCUSION}

En el periodo de observación se encontró que el 9\% de las personas que tuvieron un auto-reporte positivo para el consumo de alcohol, que es cercana a la prevalencia encontrada en el informe de alcohol y trauma en el servicio de urgencias de la Organización de Naciones Unidas y en el informe de la Organización Panamericana de la Salud, donde reportan valores entre 10-20\%.37,38 Se debe tener en cuenta que los valores de los autoreportes suelen ser más bajos que los que se encuentran cuando se realizan pruebas de detección de consumo mediante pruebas de aliento o sangre, ${ }^{39}$ y cerca de la mitad de los pacientes con alcoholemia positiva, tienen consumo positivo para otra sustancia psicotrópica como marihuana, cocaína, y opiáceos, ${ }^{37}$ los cuales no fueron objeto de evaluación para esta población.
Comparando los dos hospitales en los cuales se tomaron los datos, se encontró que el hospital público presenta 10 veces más auto-reporte positivo comparado con el privado, lo que concuerda con los patrones de consumo alcohólico en los estratos económico-sociales más bajos que suelen consumir de manera mas frecuente e intensa. ${ }^{37,38}$

Aunque el mecanismo de trauma más frecuentemente encontrado en los dos centros fue el contuso (55\%), seguido por el relacionado con eventos de transito (14\%), y los debidos a lesiones por arma blanca (13\%) y de fuego (11\%); fueron estos dos últimos los que más se asociaron con un auto-reporte de consumo de alcohol positivo. Este resultado es consistente con lo descrito en otros estudios, en donde las personas que consumen alcohol sufren mas frecuentemente lesiones violentas y relacionadas con eventos de transito, con un OR de 15.0 (95\% IC, 5.8-39.1) para lesiones intencionales y un OR de 4.2 (95\% IC, 2.7-6.5) para lesiones no intencionales, ${ }^{19,37,38,40,41}$ con tendencia al aumento con dosis de alcohol mayores. ${ }^{38}$

Otras características sociodemográficas más frecuentemente relacionadas con el consumo alcohólico en nuestro estudio fueron el ser hombre, tener entre 20 y 44 años, no tener un aseguramiento de salud, y encontrarse realizando actividades de ocio. Estas características también se han descrito en otras poblaciones, específicamente en uno realizado en 19 países de todos los continentes y con 5410 pacientes en el que se describe que el ultimo consumo se realizo entre 30-60 minutos previos al trauma, lo cual ocurre en sectores públicos, y se presenta tanto en personas con patrón de consumo ocasional como el consumidor habitual. ${ }^{37,38}$ Otro estudio encontró que la mayoría de estos pacientes se han sentido embriagados previo al evento traumático, y se encontraban festejando algún evento. ${ }^{42}$

En cuanto a la relación entre el consumo de alcohol y la severidad del trauma sufrido, a diferencia de lo reportado en la literatura, ${ }^{17,43-46}$ en nuestros pacientes la asociación con la severidad del trauma fue errático, en tanto que la relación con la severidad del deterioro fisiológico fue muy consistente.

La identificación de un grupo considerable de pacientes con probabilidad muy baja de haber sido expuestos a bebidas alcohólicas y otros grupos con alta prevalencia de consumo de alcohol permitirían focalizar los esfuerzos, permitiendo un mejor rendimiento de los recursos invertidos. Nuestros hallazgos deben ser confirmados con estudios diseñados específicamente para el efecto.

\section{REFERENCIAS}

1. Patton GC, Coffey C, Sawyer SM, Viner RM, Haller DM, Bose $\mathrm{K}$, et al. Global patterns of mortality in young people: 
a systematic analysis of population health data. Lancet 2009; 374(9693):881-892.

2. Naci H, Chisholm D, Baker TD. Distribution of road traffic deaths by road user group: a global comparison. Inj Prev 2009;15(1):55-59.

3. Rehm J, Mathers C, Popova S, Thavorncharoensap M, Teerawattananon Y, Patra J. Global burden of disease and injury and economic cost attributable to alcohol use and alcohol-use disorders. Lancet 2009;373(9682):2223-2233.

4. Bhalla K, Harrison J, Abraham J, Borse NN, Lyons R, Boufous S, et al. Data sources for improving estimates of the global burden of injuries: call for contributors. PLoS Med 20 de enero de 2009;6(1):e1-e1.

5. Gutierrez-Martinez MI, Villin RE Del, Fandiño A, Oliver RL. The evaluation of a surveillance system for violent and nonintentional injury mortality in Colombian cities. Int J Inj Contr Saf Promot 2007;14(2):77-84.

6. Posada J, Ben-Michael E, Herman A, Kahan E, Richter E. Death and injury from motor vehicle crashes in Colombia. Rev Panam Salud Publica 2000;7(2):88-91.

7. Rehm J, Gmel G, Sempos CT, Trevisan M. Alcohol-related morbidity and mortality. Alcohol research and health: the Journal of the National Institute on Alcohol Abuse and Alcoholism 2003;27(1):39-51.

8. Darke $S$. The toxicology of homicide offenders and victims: A review. Drug Alcohol Rev 2010;29(2):202-215.

9. Blomberg RD, Peck RC, Moskowitz H, Burns M, Fiorentino D. The Long Beach/Fort Lauderdale relative risk study. J Saf Res 2009;40(4):285-292.

10. Room R, Babor T, Rehm J. Alcohol and public health. Lancet 2005;365(9458):519-530

11. Herrán OF, Ardila MF, Valle U. Consumo de alcohol, riesgo de alcoholismo y alcoholismo en Bucaramanga, Colombia, 2002-2003. Colombia Médica 2005;36:158-167.

12. Herrán AM. Categories of alcohol consumers and the criteria for classification. Biomed 2009 Dec;29(4):635-646.

13. Herrán, Ardila MF BD. Test for assessing levels of alcohol consumption in Bucaramanga, Colombia: design and validation. Biomed 2008;28(1):25-37.

14. Social FES. Estudio Nacional de Salud Mental Colombia 2003. 2003. Acceso en http://onsm.ces.edu.co/uploads/ files/1243030_EstudioNacionalSM2003.pdf, Mayo de 2015.

15. Herrán OF, Ardila MF. Validity and reproducibility of two semi-quantitative alcohol frequency questionnaires for the Colombian population. Public Heal Nutr 2006;9(6):763-770.

16. Buikhuisen W, Plas-Korenhoff C, Bontekoe EHM. Alcohol and Violence. En: Moffitt T, Mednick S, editores. Biological Contributions to Crime Causation SE - 15. Springer Netherlands; 1988. p. 261-276.

17. Watt K, Purdie DM, Roche AM MR. Injury severity: role of alcohol, substance use and risk-taking. Emerg Med Australas 2006;18(2)108-117.

18. Watt K, Purdie DM, Roche AM MR. Risk of injury from acute alcohol consumption and the influence of confounders 1999;99(10):1262-1273

19. Borges G, Cherpitel C, Orozco R, Bond J, Ye Y, Macdonald S, et al. Multicentre study of acute alcohol use and non-fatal injuries: data from the WHO collaborative study on alcohol and injuries. Bull World Health Organ 2006;84(6):453-460.

20. Borges G, Cherpitel CJ, Orozco R, Bond J, Ye Y, Macdonald S GN, Stockwell T, Cremonte M, Moskalewicz J, Swiatkiewicz GPV. Acute alcohol use and the risk of non-fatal injury in sixteen countries. Addict 2006;101(7):993-1002.
21. Andelic N, Jerstad T, Sigurdardottir SS. Effects of acute substance use and pre-injury substance abuse on traumatic brain injury severity in adults admitted to a trauma centre. J Trauma Manag Outcomes 2010;4:6.

22. Desapriya E, Pike I, Raina P. Severity of alcohol-related motor vehicle crashes in British Columbia: case - control study. Int J Inj Contr Saf Promot 2006;13(2):89-94.

23. Taylor B, Irving HM, Kanteres F, Room R, Borges G, CherpitelC, et al. The more you drink, the harder you fall: A systematic review and meta-analysis of how acute alcohol consumption and injury or collision risk increase together. Drug Alcohol Depend 2010;110(1):108-116.

24. Cherpitel CJ, Bond J, Ye Y, Borges G, MacDonald S, Stockwell T, et al. Alcohol-related injury in the ER: a cross-national metaanalysis from the Emergency Room Collaborative Alcohol Analysis Project (ERCAAP). J Stud Alcohol 2003;64(5):641-649.

25. Rivara FP, Jurkovich GJ, Gurney JG, Seguin D, Fligner CL, Ries R, et al. The magnitude of acute and chronic alcohol abuse in trauma patients. Arch Surg 1993;128(8):903-907.

26. Soderstrom CA, Dischinger PC, Smith GS, Hebel JR, McDuff DR, Gorelick DA, et al. Alcoholism at the time of injury among trauma center patients: vehicular crash victims compared with other patients. Accident; analysis and prevention 1997 Nov;29(6):715-721.

27. Soderstrom CA, Dischinger PC, Smith GS, McDuff DR, Hebel JR, Gorelick DA. Psychoactive substance dependence among trauma center patients. JAMA 1992;267(20):2756-2759.

28. Babor TF, Caetano R. Evidence-based alcohol policy in the Americas: strengths, weaknesses, and future Challenges. Rev Panam Salud Publica 2005;18(4-5):327-337.

29. Shandro JR, Rivara FP, Wang J, Jurkovich GJ, Nathens AB, MacKenzie EJ. Alcohol and risk of mortality in patients with traumatic brain injury. J Trauma 2009;66(6):1584-1590.

30. Caufeild J, Singhal A, Moulton R, Brenneman F, Redelmeier D, Baker AJ. Trauma recidivism in a large urban Canadian population. J Trauma 2004;57(4):872-876.

31. Rivara FP, Koepsell TD, Jurkovich GJ, Gurney JG, Soderberg $\mathrm{R}$. The effects of alcohol abuse on readmission for trauma. JAMA 1993;270(16):1962-1964.

32. Gentilello LM, Rivara FP, Donovan DM, Jurkovich GJ, Daranciang E, Dunn CW, et al. Alcohol interventions in a trauma center as a means of reducing the risk of injury recurrence. Ann Surg 1999;230(4):473-480.

33. Gentilello LM, Ebel BE, Wickizer TM, Salkever DS, Rivara FP. Alcohol Interventions for Trauma Patients Treated in Emergency Departments and Hospitals. Ann Surg 2005; 241(4):541-550.

34. García F, Pérez A. Factores modificadores de la alcoholemia. Revista Clínica Española 1998;198:57-60.

35. Bejarano M, Rendón F. Lesiones de causa externa en menores y mayores de 18 años en un hospital colombiano. Rev Panam Salud Publica 2009;25(3):234-241.

36. Calle-Toro JS, Ordonez C, Sánchez ÁI, Sanjuan J, Badiel M, Pino L, et al. Epidemiología de Lesiones Relacionadas con Colisiones de Vehículos Motorizados en dos Centros de Referencia del Suroccidente Colombiano. Reporte del Registro Internacional de Trauma de la Sociedad P. Rev Panam Trauma 2013;16-22.

37. Ponziak, Vladimir, Paden M. Alcohol and Injury in Emergency Departments, 2007. Summary of the Report from the WHO Collaborative Study on Alcohol and Injuries. WHO 2007;1-18. 
38. Cherpitel CJ, Borges G, Giesbrecht N. Prevención de los traumatismos relacionados con el alcohol en las Américas: de los datos probatorios a la acción política 2013;1-189.

39. Skinner HA, Holt S, Sheu WJ, Israel Y. Clinical versus laboratory detection of alcohol abuse: the alcohol clinical index. Br Med J (Clin Res Ed) 1986;292(6537):1703-1708.

40. Watt K, Purdie DM, Roche AM, McClure R. Acute alcohol consumption and mechanism of injury. Journal of Studies on Alcohol 2006;67(1):14-21.

41. Borges G, Orozco R, Cremonte M, Buzi-Figlie N, Cherpitel C, Poznyak V. Alcohol and violence in the emergency department: a regional report from the WHO collaborative study on alcohol and injuries. Salud publica de Mexico 2008; 50(Suppl 1):S6-S11.

42. Cherpitel CJ, Bond J, Ye Y, Borges G, Room R, Poznyak V, et al. Multi-level analysis of causal attribution of injury to alcohol and modifying effects: Data from two international emergency room projects. Drug Alcohol Depend 2006;82(3): 258-268.

43. Blondell RD, Looney SW, Krieg CL, Spain DA. A comparison of alcohol-positive and alcohol-negative trauma patients. J Stud Alcohol 2002;63(3):380-383.

44. Cassiani CA, Mayerly Cubides Á, Varona B, Tatiana M, Marimón Trespalacios W. Alcohol and trauma: a priority public health problem. Salud Uninorte 2012;28(1): 131-149.

45. Watt K, Purdie DM, Roche AM, McClure RJ. The relationship between acute alcohol consumption and consequent injury type. Alcohol Alcohol 2005;40(4):263-268.

46. Watt K, Purdie DM, Roche AM, McClure RJ. Risk of injury from acute alcohol consumption and the influence of confounders. Addiction 2004;99(10):1262-1273. 
La ingesta de alcohol se ha asociado con certeza a la incidencia de lesiones traumáticas. Muchas sociedades ha expresado a través de su política de salud publica la preocupación por el descuidado uso del alcohol durante actividades que requieren lucidez mental. Este estudio es un ejemplo íntegro del valor de los registros de trauma dentro de la amplitud de un centro de trauma reconociéndolo como un vehículo de transformación social. Esta información del panorama demográfico de la población afectada por el consumo de alcohol por ejemplo confirma los resultados de estudios previos en donde pacientes que sufren lesiones debido a traumatismos por motoras, se caracterizan por presentar las edades de 30-49 años, llegar al hospital tarde en la noche y no usar el casco de protección. ${ }^{1,2}$

Se ha encontrado que hasta casi la mitad de las admisiones a un centro de trauma están relacionadas al consumo de alcohol y/o el uso de drogas ilícitas. Además, estudios han confirmado que un gran número de fallecidos a causa de un evento traumático esta relacionado al consumo de alcohol (mas del $40 \%)^{2}$

Desafortunadamente, pacientes con lesiones relacionadas al consumo de alcohol presentan un mayor uso de los recursos hospitalarios, con un aumento significativo en la carga financiera. Compañías aseguradoras en muchos de los estados de los Estados Unidos por ejemplo no proporcionan cobertura por lesiones relacionadas al consumo de alcohol, trasladando estos gastos a los centros de trauma y/o los proveedores. ${ }^{3}$

Una de las partes mas importantes de esta información es la evidencia de la reincidencia de los pacientes del trauma y su relación al consumo de alcohol. Los mayores valores de la reincidencia fueron encontrados en pacientes los cuales presentaban el uso de alcohol o drogas ilícitas el día que ocurrió el trauma y en víctimas de violencia interpersonal, definido como los que tuvieron heridas por arma blanca, de fuego o agresiones personales. McCoy y et al encontraron que el $25 \%$ de la población estudiada presentaba una reincidencia del trauma. La población afectada consistía de $75 \%$ varones, $36 \%$ desempleados, $26 \%$ sin seguranza y $90 \%$ solteros. ${ }^{4}$ Esta información es vital para el desarrollo de programas de prevención específicos para la comunidad en general, pero en específico a la que presenta el comportamiento no deseado.

Los autores han investigado con bastante precisión las características demográficas de la población afectada. Una de las recomendaciones es la revisión de la data para indagar el porcentaje de los pacientes que sufrieron de un evento traumático por más de una ocasión. Una parte esencial de la información es investigar el porcentaje de la población con mas alta incidencia de sufrir un trauma en relación al uso de alcohol. Es imperativo reconocer este tipo de problema social ya que el impacto en los años de vida potencialmente perdidos afecta de forma considerable el golpe económico no solo de la unidad familiar, pero de la sociedad en general.

Seria interesante conocer si los autores planifican utilizar las intervenciones breves de tipo motivacional descritas y utilizadas por la mayoría de los centros de trauma de los Estados Unidos. Gentilello et al demostró que un 27\% de todos los pacientes con lesiones son candidatos para una breve intervención relacionada al uso de alcohol. Si estas intervenciones fueran ofrecidas rutinariamente a estos pacientes a través de la nación (EU), se podría llegar a un ahorro potencial neto de 1.82 billones de dólares anualmente. ${ }^{5}$

Este tipo de estudios demográficos procedentes de los centros de trauma a través de Sur América utilizando los registros de trauma son una evidencia contundente del compromiso de estas instituciones no solamente con la comunidad y la sociedad en general, si no también con el mejoramiento del manejo del paciente politraumatizado. Exhortamos a los centros de trauma que continúen con esta misión y compromiso a través de las naciones panamericanas.

\section{REFERENCIAS}

1. Liu HT, Liang CC, Rau CS, Hsu SY, Hsieh CH. eCollection 2015.Alcohol-related hospitalizations of adult motorcycle riders. World J Emerg Surg 2015 Jan 7;10(1):2.

2. Carrasco CE, Godinho M, Berti de Azevedo Barros M, Rizoli S, Fraga GP. Fatal motorcycle crashes: a serious public health problem in Brazil. World J Emerg Surg 2012 Aug 22;7 Suppl 1:S5.

3. O'Keeffe T1, Rhee P, Shafi S, Friese RS, Gentilello LM Trauma Acute C. Alcohol use increases diagnostic testing, procedures, charges, and the risk of hospital admission: a population-based study of injured patients in the emergency department. Am J Surg 2013 Jul;206(1):16-22. 
4. McCoy AM, Como JJ, Greene G, Laskey SL, Claridge JA. A novel prospective approach to evaluate trauma recidivism: the concept of the past trauma history. The Journal of Trauma and Acute Care Surgery 2013 Jul;75(1):116-121.

5. Gentilello LM, Ebel BE, Wickizer TM, Salkever DS, Rivara FP. Alcohol interventions for trauma patients treated in emergency departments and hospitals: a cost benefit analysis. Annals of Surgery 2005 Apr;241(4):541-550.

Manuel Lorenzo

Professor, Department of Surgery Methodist Hospital, Dallas, Texas, USA 
Alcohol ingestion has been associated with an increased incidence of traumatic injuries. This association has led to the development of public health policies limiting alcohol use during activities that require mental clarity. The development of such policies is a perfect example of how vital a trauma registry is to a trauma center; the registry becomes an instrument for the trauma center to affect social transformation. The trauma registry was used to abstract data confirming previous literature showing motorcycle crashes suffered most characteristically by individuals between the ages of 30-49 years old, crashes occurring at nighttime and by those not wearing a helmet. ${ }^{1,2}$ Almost half of the admissions to a trauma center and more than $40 \%$ of traumatic fatalities are related to the use of alcohol and/or illicit drugs. ${ }^{2}$ Consequently, alcohol-related injuries result in increased use of hospital resources and a significant financial burden. A number of insurance companies in the United States, for example, do not provide coverage to patients with traumatic injuries due to alcohol use, therefore, transferring those costs to the trauma center and/or the providers. ${ }^{3}$

The authors have demonstrated with significant precision the demographic characteristics of their trauma population. There was a higher incidence of trauma recidivism in patients that used alcohol and/or illicit drugs. McCoy et al found a $25 \%$ of recidivism among their trauma population with its cohort shown to be $75 \%$ male, $36 \%$ unemployed, $26 \%$ uninsured and $90 \%$ single. ${ }^{4}$ This data is key to developing specific prevention programs for the community, allowing to target groups showing the undesirable behavior.

One of the recommendations for the authors of this manuscript is to include in their analysis the percentage of alcohol-related recidivism. It is imperative to recognize this social issue as its potential impact in the lost life-years is a considerable economic loss not only for the family unit but also for society in general.

It would be interesting to know if the authors are planning to use the brief alcohol interventions used by most of the trauma centers in the United States. Gentilello, et al showed that $27 \%$ of all the injured patients are candidates for a brief alcohol intervention. It is estimated that routine patient interventions can result in a potential net saving of $\$ 1.82$ billion dollars annually. ${ }^{5}$

Use of trauma registries to produce demographic studies by South American trauma centers is strong evidence of their commitment not only to care of the trauma patient but to the community and society as a whole. We encourage such trauma centers to continue with this mission and commitment across the panamerican nations.

\section{REFERENCES}

1. Liu HT, Liang CC, Rau CS, Hsu SY, Hsieh CH. eCollection 2015.Alcohol-related hospitalizations of adult motorcycle riders. World J Emerg Surg 2015 Jan 7;10(1):2. doi: 10.1186/1749-7922-10-2.

2. Carrasco CE, Godinho M, Berti de Azevedo Barros M, Rizoli S, Fraga GP. Fatal motorcycle crashes: a serious public health problem in Brazil. World J Emerg Surg 2012 Aug 22;7 Suppl 1:S5. doi: 10.1186/1749-7922-7-S1-S5. Epub 2012 Aug 22.

3. O'Keeffe T1, Rhee P, Shafi S, Friese RS, Gentilello LM Trauma Acute C. Alcohol use increases diagnostic testing, procedures, charges, and the risk of hospital admission: a population-based study of injured patients in the emergency department. Am J Surg 2013 Jul;206(1):16-22. doi: 10.1016/j.amjsurg.2012.08.014. Epub 2013 Apr 3.

4. McCoy AM, Como JJ, Greene G, Laskey SL, Claridge JA. A novel prospective approach to evaluate trauma recidivism: the concept of the past trauma history. The Journal of Trauma and Acute Care Surgery 2013 Jul;75(1):116-121.

5. Gentilello LM, Ebel BE, Wickizer TM, Salkever DS, Rivara FP. Alcohol interventions for trauma patients treated in emergency departments and hospitals: a cost benefit analysis. Annals of Surgery 2005 Apr;241(4):541-550. 